\title{
TyrR, the regulator of aromatic amino acid metabolism, is required for mice infection of Yersinia pestis
}

\author{
Zhongliang Deng ${ }^{1,2+}$, Zizhong Liu ${ }^{2+}$, Junming $\mathrm{He}^{2}$, Jing Wang ${ }^{2,3}$, Yanfeng Yan ${ }^{2}$, Xiaoyi Wang ${ }^{2}$, \\ Yujun Cui ${ }^{2}$, Yujing $\mathrm{Bi}^{2}$, Zongmin $\mathrm{Du}^{2}$, Yajun Song ${ }^{2}$, Ruifu $\mathrm{Yang}^{2 *}$ and Yanping Han ${ }^{2 *}$ \\ Department of Sanitary Inspection, School of Public Health, University of South China, Hengyang, China \\ 2 State Key Laboratory of Pathogen and Biosecurity, Beijing Institute of Microbiology and Epidemiology, Beijing, China \\ ${ }^{3}$ Animal Husbandry Base Teaching and Research Section, College of Animal Science and Technology, Hebei North University, Zhangjiakou, China
}

Edited by:

Yi-Cheng Sun, Chinese Academy of Medical Sciences and Peking Union Medical College, China

Reviewed by:

Yan Ling, Beijing Institute of Biotechnology, China

Christopher Francis Bosio, National Institutes of Health, USA

${ }^{*}$ Correspondence:

Ruifu Yang and Yanping Han, No. 20 Dongdajie, Fengtai, Beijing 100071,

China

e-mail: ruifuyang@gmail.com;

yanpinghan@gmail.com

these authors have contributed equally to this work.
Yersinia pestis, the causative agent of plague, poses a serious health threat to rodents and human beings. TyrR is a transcriptional regulator (TyrR) that controls the metabolism of aromatic amino acids in Escherichia coli. In this paper, TyrR played an important role in $Y$. pestis virulence. Inactivation of tyrR did not seem to affect the in vitro growth of this organism, but resulted in at least 10,000-fold attenuation compared with the wild-type (WT) strain upon subcutaneous infection to mice. In addition, loads of tyrR mutant within mice livers and spleens significantly decreased compared with the WT strain. Transcriptome analysis revealed that TyrR, directly or indirectly, regulated 29 genes encoded on $Y$. pestis chromosome or plasmids under in vitro growth condition. Similar to the regulatory function of this protein in $E$. coli, five aromatic-pathway genes (aroF-tyrA, aroP, aroL, and tyrP) were significantly reduced upon deletion of the tyrR gene. Two genes ( $g / n L$ and $g / n G)$ that encode sensory histidine kinase and regulator in a two-component regulatory system involved in nitrogen assimilation were downregulated in the tyrR mutant. Several genes encoding type III secretion proteins were transcribed by 2.0-4.2-fold in a tyrR mutant relative to the WT strain. Interestingly, the acid-stressed genes, hdeB and hdeD, were downregulated, and such downregulation partly accounted for the decrease in tolerance of the tyrR mutant under acidic conditions. In conclusion, regulation of TyrR in $Y$. pestis is similar to, but distinct from, that in E. coli. TyrR is a metabolic virulence determinant in $Y$. pestis that is important for extracellular survival and/or proliferation.

Keywords: Yersinia pestis, TyrR, aromatic amino acid metabolism, pathogenesis

\section{INTRODUCTION}

Yersinia pestis can cause fatal infections in rodents and humans and is usually transmitted by flea biting (Perry and Fetherston, 1997). A few virulence determinants have been defined based on their contributions to flea transmission colonization, invasion, intracellular growth or extracellular proliferation (Charnetzky and Shuford, 1985; Lindler et al., 1990; Galyov et al., 1991; Lahteenmaki et al., 1998). Numerous efforts to determine the mechanism of Yersinia pathogenesis are mainly focused on a few previously established virulence determinants (Perry and Fetherston, 1997). Many other genes encoding two-component systems or global transcriptional regulators have been proven to be implicated in the regulatory networks involved in Y. pestis pathogenicity (Oyston et al., 2000; Cathelyn et al., 2006; Zhan et al., 2008; Geng et al., 2009). More than 30 genes responsible for critical metabolic pathways functioned during $Y$. pestis fitness in vivo (Palace et al., 2014). Y. pestis lacking $\sim 47-\mathrm{kb}$ DNA fragment that contains more than 40 genes was obtained by our laboratory. Surprisingly, we observed the significantly attenuated virulence of this mutant via subcutaneous infection in mice. This observation prompted us to investigate which gene or operon was responsible for such attenuation. After careful tracing via gene knockout within the 47-kb region, a transcriptional regulator (TyrR) was confirmed to be mainly responsible for the virulence phenotype. A number of DNA-binding regulators could control gene expression common to many bacterial species. TyrR is responsible for aromatic amino acids metabolism in Escherichia coli, which is evolutionarily acquired and is only present in $\gamma$ proteobacteria (Panina et al., 2001; Song et al., 2005). Subsequent evidence showed that the recruitment of structure genes by TyrR is dynamic and is evolving further (Pittard et al., 2005). Here we showed that TyrR is required for $Y$. pestis pathogenesis and extracellular survival/proliferation. Transcriptome analysis demonstrated that the regulation of TyrR in Y. pestis is similar to, but distinct from, that in E. coli.

\section{EXPERIMENTAL PROCEDURE BACTERIAL STRAINS}

Y. pestis wild-type (WT) strain 201, 47-kb fragment and tyrR deletion mutant were used in this study. Strain 201 was isolated from Microtus brandti in Inner Mongolia, China. Strain 201 is supposed to be avirulent to humans, but highly lethal to mice (Fan et al., 
1995). The deletion mutant of $Y$. pestis was constructed by replacing the entire target gene with the kan cassette by using $\lambda$-Red homologos recombination. To obtain a strain in which $\Delta t y r R$ is complemented, plasmid pACYC184, which contains a PCR fragment covering a region from $400 \mathrm{bp}$ fragment upstream to $100 \mathrm{bp}$ downstream of the tyrR gene, was introduced into $Y$. pestis $\Delta t y r R$.

\section{DETERMINATION OF BACTERIAL GROWTH CURVES IN VITRO}

$Y$. pestis strains were grown in $\mathrm{LB}$ medium at $26^{\circ} \mathrm{C}$ to exponential phase $\left(\mathrm{OD}_{620} \approx 1.0\right)$. The bacterial cultures were diluted 1:20 in $\mathrm{LB}$ medium with the indicated $\mathrm{pH}$ value and incubated at $26^{\circ} \mathrm{C}$. Bacterial growth was monitored by measuring absorbance at $\mathrm{OD}_{620}$. The experiments were performed in three independent cultures. Results were expressed as the mean percentage \pm standard deviation from the three independent experiments.

\section{MOUSE INFECTION}

$Y$. pestis strains were grown in brain-heart infusion (BHI) broth at $26^{\circ} \mathrm{C}$ to $\mathrm{OD}_{620} \approx 1.0$. Bacterial cells were harvested by centrifugation, washed twice and resuspended in phosphate-buffered saline (PBS). Groups of five or six female BALB/c mice (6-weeks-old) were injected subcutaneously with serial dilutions of bacterial cultures. Mortality was recorded daily for $14 \mathrm{~d}$. The 50\% lethal dose $\left(\mathrm{LD}_{50}\right)$ was calculated by the Reed-Muench equation (Reed and Muench, 1938).

To monitor bacterial growth dynamics in vivo, Y. pestis WT strain 201 and the tyrR deletion mutant grown to exponential phase were washed and diluted to $600 \mathrm{CFU} / \mathrm{mL}$ in PBS. A 1:1 mixture $(100 \mu \mathrm{L})$ of the two bacterial strains was used to infect eight BALB/c mice intravenously. For competitive index (CI) determination, the infected mice were sacrificed at 48 and $72 \mathrm{~h}$ postinfection, and livers and spleens were removed for homogenization. Bacterial loads per organ were determined by calculating the number of viable bacteira of the resulting homogenates plated on Hottinger agar with and without kanamycin. The CI value was calculated as the ratio of the number of mutant/WT bacteria recovered. The data were analyzed by Student's $t$-test, with $P<$ 0.05 considered statistically significant. All mouse experiments were carried out according to the Guidelines for the Welfare and Ethics of Laboratory Animals of Beijing.

\section{TRANSCRIPTION ANALYSIS BY USING RNA-SEQ AND REAL-TIME PCR}

$Y$. pestis WT strain 201 and the $\Delta t y r R$ mutant were grown at $26^{\circ} \mathrm{C}$ in $\mathrm{BHI}$ medium to middle exponential phase and then transferred to $37^{\circ} \mathrm{C}$ for $3 \mathrm{~h}$. Total RNA was extracted using the TRIzol Reagent according to the manufacturer's protocol (Invitrogen). These two RNA samples were subjected to cDNA library construction and deep sequencing performed by LC Sciences LLC, USA, as previously described (Yan et al., 2013). RPKM was used to estimate the expression levels of mRNA transcripts encoded by Y. pestis chromosome and plasmids. Those genes with cDNA coverage below 20 in both samples were removed from further analysis. The fold change of mRNA expression levels between WT and tyrR mutant were calculated. Two-fold was used as threshold to determine the differentially regulated genes.

For quantitative RT-PCR, cDNA was generated using $5 \mu \mathrm{g}$ of total RNA and $3 \mu \mathrm{g}$ of random hexamer primers with the
Superscript II system. All primer pairs produced a 150-200 nt amplicon when $Y$. pestis genomic DNA was used as the template for PCR. Real-time PCR was performed in duplicate for each RNA preparation using the LightCycler system (Roche) with an appropriate dilution of cDNA as a template. Based on the standard curve of $16 \mathrm{~S}$ rRNA, the relative mRNA level was determined by calculating the threshold cycle $(\Delta \mathrm{Ct})$ of each gene by the classic $\Delta \mathrm{Ct}$ method. Quantification of $16 \mathrm{~S}$ rRNA was also used to normalize the values of all the other genes in the RT-PCR experiment.

\section{MEASUREMENT OF SECRETED LCrV PROTEIN BY USING WESTERN BLOTTING}

$Y$. pestis WT strain, the $\Delta t y r R$ mutant and the tyrR complementary strain were grown at $26^{\circ} \mathrm{C}$ in $\mathrm{TMH}$ medium without calcium to $\mathrm{OD}_{620}=1.0$ and then transferred to $37^{\circ} \mathrm{C}$ for $3 \mathrm{~h}$. Secreted proteins from bacterial supernatants were precipitated with trichloroacetic acid (TCA). Proteins from equal amount of bacteria were separated on SDS-PAGE and immunoblotted with LcrV polyclonal antibody followed by detection using the Odyssey Infrared Imaging System.

\section{RESULTS}

\section{TYrR IS REOUIRED FOR $Y$. PESTIS INFECTION UPON SUBCUTANEOUS INOCULATION TO MICE}

We observed the strong attenuation of a deletion mutant of $\sim 47$ $\mathrm{kb}$ DNA fragment containing more than 40 genes in $\mathrm{BALB} / \mathrm{c}$ mice upon subcutaneous inoculation. To trace which gene(s) or operon is responsible for the attenuated phenotype, DNA fragments within the $47-\mathrm{kb}$ region were knockout one by one from the WT strain. The resulting mutants were subject to mice infection, respectively. Finally, a $\sim 8$-kb fragment containing tyrR and its flanking sequences were confirmed to be the main reason for the virulence phenotype. Therefore, we decided to assess the roles of TyrR protein in Y. pestis pathogenesis. Mice were infected subcutaneously (s.c.) with increasing numbers of WT, tyrR mutant, and complementary strain to estimate the virulence by calculating $\mathrm{LD}_{50}$. The $\mathrm{LD}_{50}$ of both the WT strain 201 and the tyrR complementary strain was, $<8 \mathrm{CFU}$, but up to about $8 \times 10^{4}$ cells of the tyrR mutant was not lethal to s.c. inoculated mice (Figure 1). The observation suggested that the significantly attenuated virulence of the tyrR mutant is due to the lack of TyrR protein rather than to polar effects caused by the insertion of a kanamycin resistance cassette.

\section{TyrR CONTRIBUTES TO GROWTH DYNAMICS IN VIVO}

Growth of the tyrR mutant was not retarded relative to that of WT strain under the condition used in vitro (Figure 2), indicating that the mutations do not cause any defect in the growth ability. Therefore, the differences in virulence could be due to the specific involvement of this protein under in vivo conditions. We next determine the kinetics of in vivo growth to examine the fitness of $Y$. pestis WT and tyrR mutant. CI assays were performed by intravenously inoculating the bacterial mixture into mice. The results showed that load burden of WT strain can achieved $10^{7} \mathrm{CFU}$ in the spleen or liver after infection for 48 and $72 \mathrm{~h}$. However, compared with the WT strain, a relatively lower amount bacteria of 


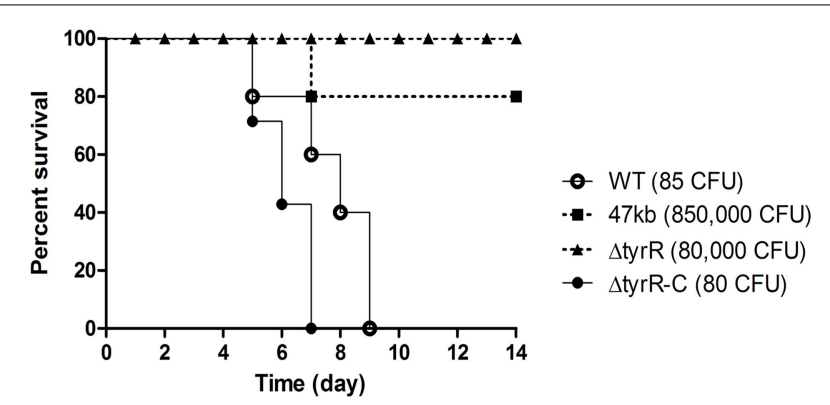

FIGURE 1 | Effect of the tyrR gene on $\boldsymbol{Y}$. pestis pathogenesis in mice. Groups of five or seven BALB/c mice were inoculated by subcutaneous injection with appropriated dose of $Y$. pestis strain 201 (open circles), 47-kb mutant (filled diamonds), tyrR (filled triangles) or tyrR complementary strain (filled circles). Mice mortality was monitored for $14 \mathrm{~d}$.

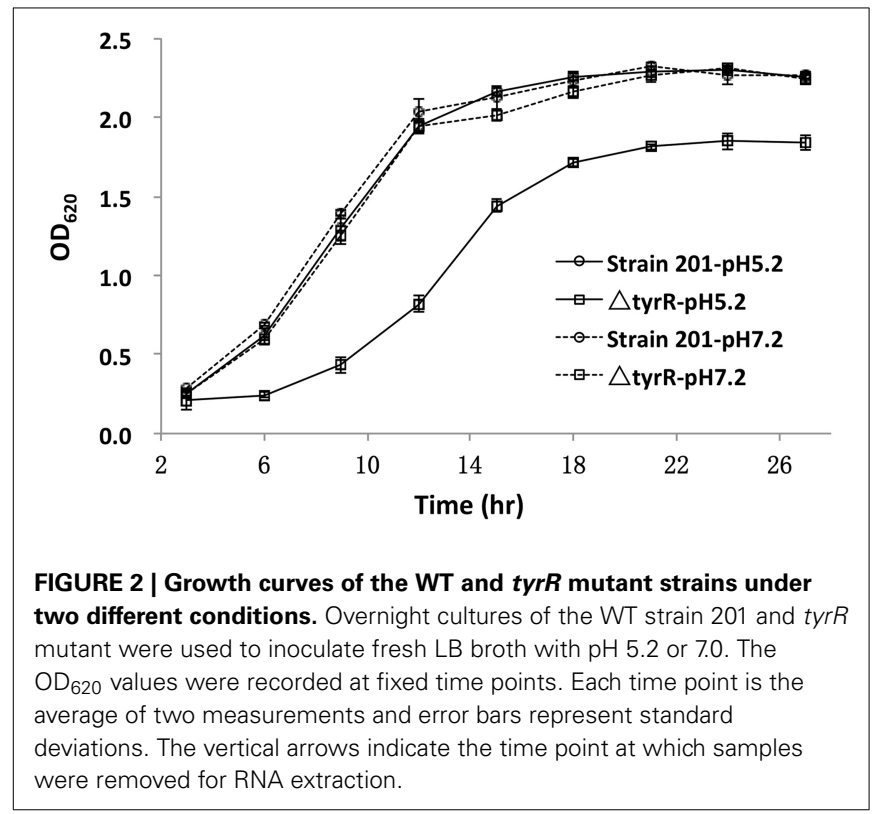

the $t y r R$ mutant strain was recovered from the spleen or liver. The mean CI values in the spleens were 0.043 at $48 \mathrm{~h}$ and 0.003 at $72 \mathrm{~h}$ (Figure 3A), and similar CI values were obtained in the livers $(0.017$ at $48 \mathrm{~h}$ and 0.005 at $72 \mathrm{~h}$ ) (Figure 3B). Clearly, the $t y r R$ mutant was nearly overwhelmed by the population of WT Y. pestis in vivo, indicating that this mutant was much less competitive in vivo than its parental strain.

\section{IDENTIFICATION OF DIFFERENTIAL GENES REGULATED BY TyrR}

To obtain a representative image of TyrR protein in affecting gene expression throughout growth, we recovered RNA from cultures in vitro at different time points to determine the optimal time points. The abundance of the $t y r R$ transcript was measured by qPCR (data not shown). The bacterial culture grown in BHI at $26^{\circ} \mathrm{C}$ for $9 \mathrm{~h}$ and then transferred to $37^{\circ} \mathrm{C}$ for $3 \mathrm{~h}$ was chosen for RNA recovery for RNA sequencing.

In total, 29 genes were shown differentially expressed between WT and tyrR mutant strain (Table 1). Of these genes, 11 were
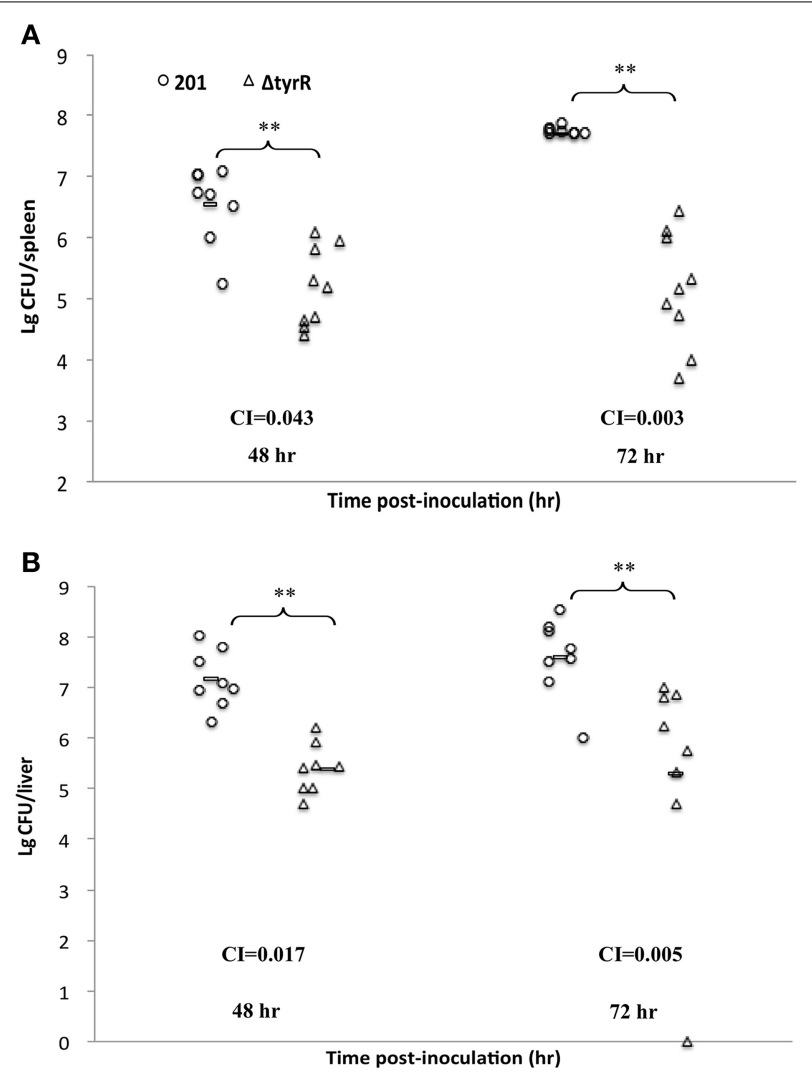

FIGURE 3 | Growth dynamics of $\boldsymbol{Y}$. pestis upon inoculation by intraveneous route. Groups of eight mice were inoculated intravenously with bacterial mixture of $Y$. pestis strain 201 and the tyrR mutant. Bacterial loads of WT strain (open circles) and tyr $R$ mutant (open triangles) in spleen (A) and liver (B) were determined at $48 \mathrm{~h}$ and $72 \mathrm{~h}$ post-inoculation. Horizontal bars indicate geometric means. The indicated $P$ values were determined using the Student's t-test.

upregulated and 18 were downregulated in tyrR mutant compared with the WT strain. Intriguingly, approximately $45 \%$ (13/29) of the differentially regulated genes were derived from three plasmids (pCD1, pMT1, and pCRY), thereby suggesting that the laterally acquired genetic elements might be recruited to TyrR regulon during the process of evolution. Of which, five genes encoding type III secretion proteins in plasmid pCD1 $(y s c B, y s c N$, $y s c P$, sycE, and $l c r V$ ) were downregulated 2.0-4.2- fold in $t y r R$ mutant relative to WT strain.

Eight genes were selected for qPCR analysis to validate the RNA-seq data. A high correlation $\left(R^{2}=0.988\right)$ was observed between expression values obtained by RNA-seq and qPCR (Figure 4). Aromatic-pathway genes were confirmed to be regulated by TyrR in E. coli (Pittard et al., 2005). As expected, the aroF-tyrA operon responsible for aromatic biosynthesis and aroL, aroP and tyrP for aromatic transport were most strongly upregulated. Two genes responsible for acid stress, $h d e B$ and $h d e D$, were confirmed to be downregulated upon deletion of tyrR gene, and this finding was consistent with the observation on the compromised tolerance to acid stress in in vitro assays (Figure 1). 
Table 1 | List of differentially regulated genes in tyrR mutant compared to WT strain.

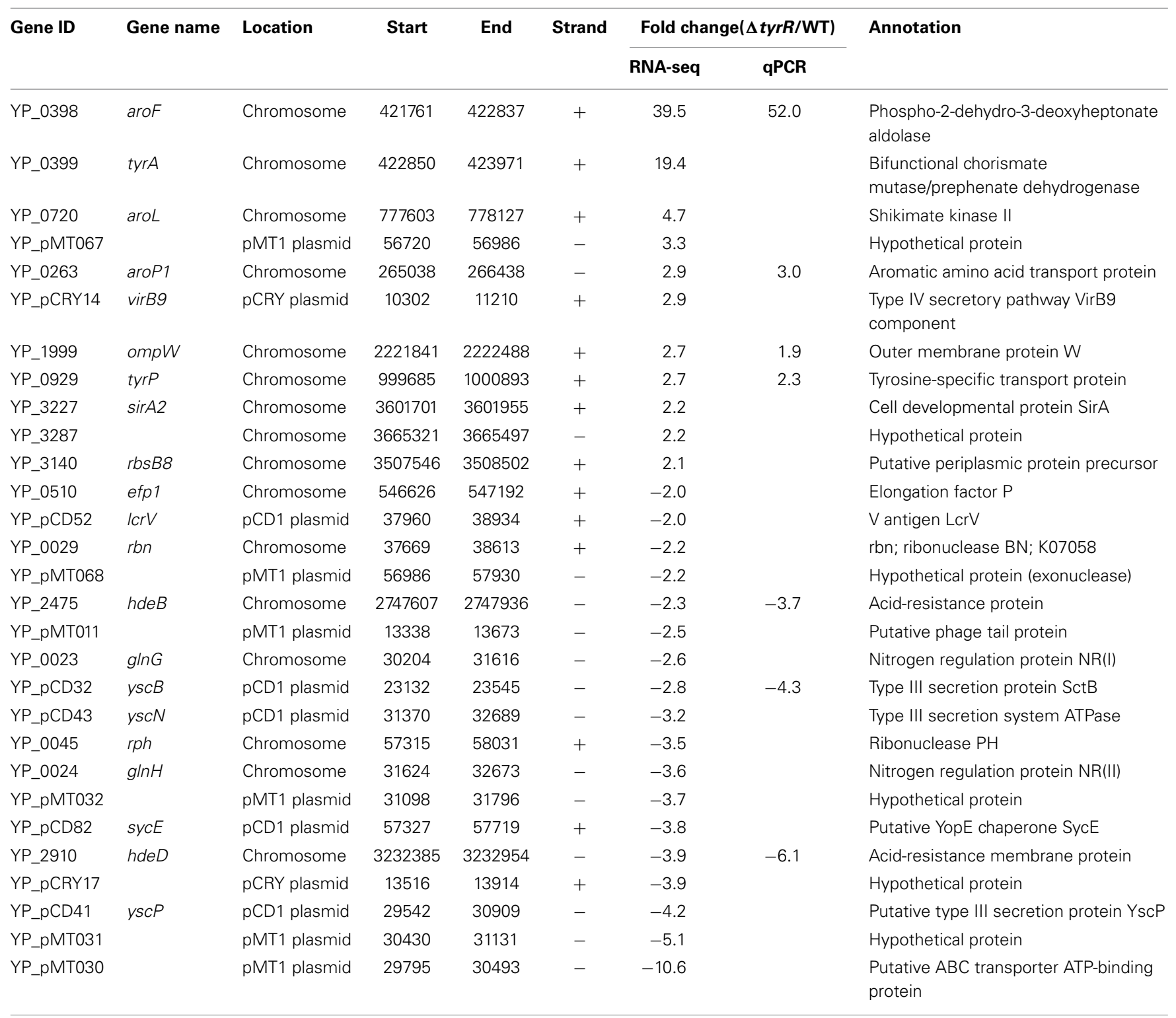

\section{USE OF CONSERVED TYRR MOTIF TO IDENTIFY DIRECT TyrR -REGULATED GENES}

DNA-binding regulator usually regulates its target genes by directly binding to a dyad DNA consensus sequence. To predict which genes identified by RNA-seq might be under the direct control of TyrR, we extracted the putative TyrR-binding motif sequence (TGTAAA- $\mathrm{N}_{6}$-TTTACA) from RegulonDB and conducted a research in the upstream $300 \mathrm{nt}$ of the differentially regulated genes identified by RNA-seq experiment. Four genes were identified as candidates for direct TyrR regulation. As expected, conserved DNA motifs were found in the promoter region of three known aromatic-pathway transcripts ( $a r o F, t y r P$, and aroP1). Another candidate targeted by TyrR is the $h d e D$ gene, which encodes an acid stress membrane protein, because TyrR box was found $\sim 140 \mathrm{nt}$ upstream of the translational start site of $h d e D$.

\section{SYNTHESIS OF SECRETED V ANTIGEN NOT INFLUENCED BY TyrR IN Y. PESTIS}

$Y$. pestis LcrV is secreted via T3SS machinery during infection and can be exploited as a protective antigen known as $\mathrm{V}$ antigen (Skrzypek and Straley, 1995). Abrogation of LcrV expression render Y. pestis avirulent (Burrows, 1956). Since LcrV protein is responsible for extracellular survival and dissemination in $Y$. pestis, the down-regulation of $\operatorname{lcr} V$ might lead to the reduced capability of organ colonization. $Y$. pestis $\mathrm{V}$ antigen is maximally expressed and secreted under calcium-restricted conditions at $37^{\circ} \mathrm{C}$ in vitro. Therefore, we compared the levels of secreted $\mathrm{V}$ antigen synthesis by using Western blot method in WT and tyrR mutant grown in calcium-deficiency TMH medium at $37^{\circ} \mathrm{C}$. Unexpectedly, the results showed that the expression of $\mathrm{V}$ antigen was not obviously changed upon the deletion of tyrR (Supplementary Figure 1). TyrR might have 


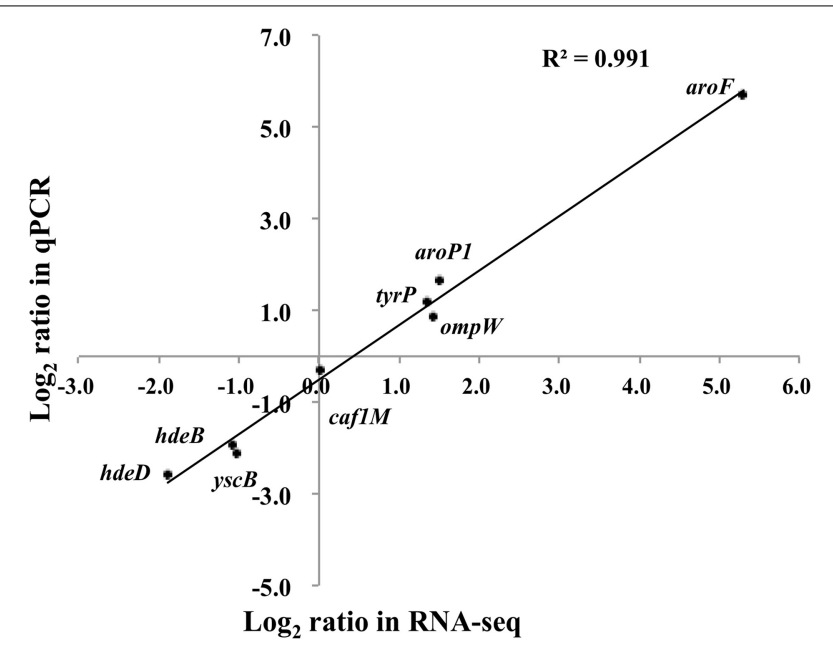

FIGURE 4 | Transcription measurements of eight genes were chosen for quantitative RT-PCR validation. The real-time PCR $\log _{2}$ values were plotted against the RNA-seq data log2 values. The coefficient of determination $\left(R^{2}\right)$ for comparison of the two datasets is 0.991 .

failed to affect $\mathrm{LcrV}$ expression at least in $Y$. pestis grown in vitro.

\section{DISCUSSION}

Aromatic amino acid biosynthesis is required for the intracellular replication of Listeria monocytogenes and Shigella flexneri (Cersini et al., 1998; Stritzker et al., 2004). Coincidently, two aromaticpathway genes (aroE and aroA) play a crucial role in Y. pestis fitness in deep tissues during infection (Palace et al., 2014). $Y$. pestis lacking tyrR was significantly less virulent than the WT strain, and complementation of $t y r R$ restored WT virulence in the deletion strain, thereby suggesting that TyrR plays an important role in Y. pestis virulence. The regulation of aromatic amino acid metabolism has been assigned to the transcription factor, TyrR. This is the first report to demonstrate the effect of this protein on Y. pestis pathogenesis. TyrR is not required for growth in vitro, but is required for full virulence of $Y$. pestis. Whether the availability of aromatic amino acids in vivo might have an effect during the process of infection unknown. Intriguingly, we observed that tyrR mutant efficiently protects mice against $Y$. pestis infection as an attenuated strain (data unpublished). The potential of TyrR as a new therapeutic target is worth exploring.

When $Y$. pestis enters invasion sites, most of the bacteria are engulfed and killed by the polymorphonuclear leukocytes (PMNs) that are attracted to these invasion sites. However, a few bacilli survive and proliferate within phagolysosomes of tissue macrophages during the initial infection stage and are then released and to elicit the systematic infection (Perry and Fetherston, 1997). The growth defect of Y. pestis tyrR mutant in BHI with pH 5.2 (Figure 1) was a hint that TyrR might be associated with intracellular replication, because acidic $\mathrm{pH}$ is a hypothesized prevailing condition in phagolysosomal microenvironments.

Based on the results of RNA-seq-based transcriptional profiling, less than $1 \%$ of $Y$. pestis genes were affected by TyrR, thereby indicating that this protein is likely to function as a local regulator. However, it was unexpected that a regulatory defect in a minority of non-essential genes has as much effect on pathogenesis in vivo as the removal of a single critical virulence factor. We supposed that the regulation of T3SS encoded by PCD1 plasmid might partially account for the virulence variations between WT and tyrR-deletion strains. However, the $\mathrm{PCD} 1$ plasmid-encoded genes are downregulated ranging from 2.0 to 4.2 fold, which are generally much lower than that for the aromatic amino acid metabolism genes $(2.7 \sim 39.5$ fold $)$. In addition, no predicted TyrR-binding sites exist in the regulated genes of T3SS, indicating perhaps TyrR weakly affects transcription of these genes due to lacking exact DNA binding motifs on these plasmids. Thus, we speculated that regulation of T3SS by TyrR is likely to be indirect.

\section{ACKNOWLEDGMENTS}

This study was funded by the National Natural Science Foundation of China (31171248) and the National Basic Research Program of China (2014CB7444405).

\section{SUPPLEMENTARY MATERIAL}

The Supplementary Material for this article can be found online at: http://www.frontiersin.org/journal/10.3389/fmicb.2015. 00110/abstract

\section{REFERENCES}

Burrows, T. W. (1956). An antigen determining virulence in Pasteurella pestis. Nature 177, 426-7. doi: 10.1038/177426b0

Cathelyn, J. S., Crosby, S. D., Lathem, W. W., Goldman, W. E., and Miller, V. L. (2006). RovA, a global regulator of Yersinia pestis, specifically required for bubonic plague. Proc. Natl. Acad. Sci. U.S.A. 103, 13514-13519. doi: 10.1073/pnas.0603456103

Cersini, A., Salvia, A. M., and Bernardini, M. L. (1998). Intracellular multiplication and virulence of Shigella flexneri auxotrophic mutants. Infect. Immun. 66, 549-557.

Charnetzky, W. T., and Shuford, W. W. (1985). Survival and growth of Yersinia pestis within macrophages and an effect of the loss of the 47-megadalton plasmid on growth in macrophages. Infect. Immun. 47, 234-241.

Fan, Z., Luo, Y., Wang, S., Jin, L., Zhou, X., Liu, J., et al. (1995). Microtus brandti plague in the Xilin Gol Grassland was inoffensive to humans. Chin. J. Control Endem. Dis. 10, 56-7.

Galyov, E. E., Karlishev, A. V., Chernovskaya, T. V., Dolgikh, D. A., Smirnov, O., Volkovoy, K. I., et al. (1991). Expression of the envelope antigen F1 of Yersinia pestis is mediated by the product of caf1M gene having homology with the chaperone protein PapD of Escherichia coli. FEBS Lett. 286, 79-82. doi: 10.1016/0014-5793(91)80945-Y

Geng, J., Song, Y., Yang, L., Feng, Y., Qiu, Y., Li, G., et al. (2009). Involvement of the post-transcriptional regulator $\mathrm{Hfq}$ in Yersinia pestis virulence. PLoS ONE 4:e6213. doi: 10.1371/journal.pone.0006213

Lahteenmaki, K., Virkola, R., Saren, A., Emody, L., and Korhonen, T. K. (1998). Expression of plasminogen activator pla of Yersinia pestis enhances bacterial attachment to the mammalian extracellular matrix. Infect. Immun. 66, 5755-5762.

Lindler, L. E., Klempner, M. S., and Straley, S. C. (1990). Yersinia pestis pH 6 antigen: genetic, biochemical, and virulence characterization of a protein involved in the pathogenesis of bubonic plague. Infect. Immun. 58, 2569-2577.

Oyston, P. C., Dorrell, N., Williams, K., Li, S. R., Green, M., Titball, R. W., et al. (2000). The response regulator PhoP is important for survival under conditions of macrophage-induced stress and virulence in Yersinia pestis. Infect. Immun. 68, 3419-3425. doi: 10.1128/IAI.68.6.3419-3425.2000

Palace, S. G., Proulx, M. K., Lu, S., Baker, R. E., and Goguen, J. D. (2014). Genomewide mutant fitness profiling identifies nutritional requirements for optimal 
growth of Yersinia pestis in deep tissue. Am. Soc. Microbiol. 5:e01385-14. doi: 10.1128/mBio.01385-14

Panina, E. M., Vitreschak, A. G., Mironov, A. A., and Gelfand, M. S. (2001). Regulation of aromatic amino acid biosynthesis in gamma-proteobacteria. J. Mol. Microbiol. Biotechnol. 3, 529-543.

Perry, R. D., and Fetherston, J. D. (1997). Yersinia pestis-etiologic agent of plague. Clin. Microbiol. Rev. 10, 35-66.

Pittard, J., Camakaris, H., and Yang, J. (2005). The TyrR regulon. Mol. Microbiol. 55, 16-26. doi: 10.1111/j.1365-2958.2004.04385.x

Reed, L. J., and Muench, H. (1938). A simple method for estimating fifty percent endpoints. Am. J. Hygiene. 27, 493-497.

Skrzypek, E., and Straley, S. C. (1995). Differential effects of deletions in lcrV on secretion of $\mathrm{V}$ antigen, regulation of the low-Ca2+ response, and virulence of Yersinia pestis. J. Bacteriol. 177, 2530-2542.

Song, J., Bonner, C. A., Wolinsky, M., and Jensen, R. A. (2005). The TyrA family of aromatic-pathway dehydrogenases in phylogenetic context. BMC Biol. 3:13. doi: 10.1186/1741-7007-3-13

Stritzker, J., Janda, J., Schoen, C., Taupp, M., Pilgrim, S., Gentschev, I., et al. (2004). Growth, virulence, and immunogenicity of Listeria monocytogenes aro mutants. Infect. Immun. 72, 5622-5629. doi: 10.1128/IAI.72.10.5622-56 29.2004

Yan, Y., Su, S., Meng, X., Ji, X., Qu, Y., Liu, Z., et al. (2013). Determination of sRNA Expressions by RNA-seq in Yersinia pestis Grown In Vitro and during Infection. PLoS ONE 8:e74495. doi: 10.1371/journal.pone.0074495
Zhan, L., Han, Y., Yang, L., Geng, J., Li, Y., Gao, H., et al. (2008). The cyclic AMP receptor protein, CRP, is required for both virulence and expression of the minimal CRP regulon in Yersinia pestis biovar microtus. Infect. Immun. 76, 5028-5037. doi: 10.1128/IAI.00370-08

Conflict of Interest Statement: The authors declare that the research was conducted in the absence of any commercial or financial relationships that could be construed as a potential conflict of interest.

Received: 17 December 2014; accepted: 29 January 2015; published online: 12 February 2015.

Citation: Deng Z, Liu Z, He J, Wang J, Yan Y, Wang X, Cui Y, Bi Y, Du Z, Song Y, Yang $R$ and Han $Y$ (2015) TyrR, the regulator of aromatic amino acid metabolism, is required for mice infection of Yersinia pestis. Front. Microbiol. 6:110. doi: 10.3389/ fmicb.2015.00110

This article was submitted to Food Microbiology, a section of the journal Frontiers in Microbiology.

Copyright (C) 2015 Deng, Liu, He, Wang, Yan, Wang, Cui, Bi, Du, Song, Yang and Han. This is an open-access article distributed under the terms of the Creative Commons Attribution License (CC BY). The use, distribution or reproduction in other forums is permitted, provided the original author(s) or licensor are credited and that the original publication in this journal is cited, in accordance with accepted academic practice. No use, distribution or reproduction is permitted which does not comply with these terms. 